\title{
A rare case of implant displacement to the contralateral side after gluteal augmentation
}

\author{
Juan Dario Alviar Rueda ${ }^{1}$, Audrey Jose Miranda-Diaz ${ }^{2}$, Adriana Gonzalez Cely ${ }^{3}$, \\ Diana Carolina Navarro Leon ${ }^{3}$ \\ ${ }^{1}$ Department of Plastic Surgery, Hospital Universitario de Santander, Universidad Industrial de Santander, Bucaramanga; ${ }^{2}$ Health Faculty, \\ Universidad Santiago de Cali, Cali; ${ }^{3}$ Department of Plastic Surgery, Universidad Industrial de Santander, Bucaramanga, Colombia
}

In this report, we present a rare case of solid silicone implant displacement to the contralateral side after aesthetic gluteal augmentation, a phenomenon that has never been reported before in the literature. A 29-year-old woman with a history of gluteal augmentation 9 months previously and soft tissue infection presented for a consultation due to 3 days of sudden progressive pain in the right gluteus with erythema and edema, without a history of trauma. Displacement of the left gluteal implant to the right gluteal pocket was shown by magnetic resonance imaging. Because the patient refused implant removal, the decision was made to perform capsulotomy, to reconstruct the gluteal pockets, and to preserve the implants. The patient showed a satisfactory early and late postoperative course. Possible causes of this complication include poor surgical technique, with insufficient tissue preservation to keep the pockets apart, and the presence of seroma or hematoma that favored an infectious process, thereby leading to deterioration of the dissected soft tissues with dehiscence of the wound favoring the displacement of the implant.

Keywords Buttocks / Silicone / Surgery, plastic / Postoperative complications

Received: February 28, 2019 • Revised: October 5, $2019 \bullet$ Accepted: October 18, 2019

pISSN: 2234-6163・ elSSN: 2234-6171 • https://doi.org/10.5999/aps.2019.00325・Arch Plast Surg 2020;47:360-364

\author{
Correspondence: \\ Audrey Jose Miranda-Diaz \\ Health Faculty, Universidad Santiago \\ de Cali, Calle 5 \# 62-00, Cali, \\ Colombia \\ Tel: +57-2-518-3000 \\ E-mail: audrey.miranda00@usc.edu.co
}

\section{INTRODUCTION}

Gluteal augmentation, which initially became a widespread phenomenon in Central and South America, has become more common worldwide in the last decades, increasing in frequency by more than $50 \%$, gaining popularity as an aesthetic surgical procedure, and representing a major challenge for surgeons in terms of surgical technique, results, and patient satisfaction $[1,2]$.

The surgical techniques for gluteal augmentation include gluteoplasty with solid silicone implants (subfascial or intramuscu- lar), which has the advantage of good and long-lasting results; the use of implants is an alternative for patients with a fat-poor donor site, in whom autologous fat grafting cannot be performed [2-5].

Case series have reported complication rates of up to $30 \%$ after gluteal augmentation [1]. These complications can be classified as minor or major. Minor complications include changes and irregularities in the visual aspect of the gluteus, seroma development, and palpable and visible implants, while major complications include infection, wound dehiscence, capsule contracture, extrusion, implant loss, severe ptosis, and displacement of the 
implant $[4,5]$. The most frequent complications associated with this technique are related to the surgical wound, primarily including wound dehiscence and seroma development. Additionally, few reports in the literature have dealt with specific complications, and there is no concrete information on the frequency of complications associated with this procedure that would enable an adequate comparison with our case.

The other surgical option for gluteal augmentation is lipoinjection, which has the advantages of a greater ease of shaping, a natural appearance, and ease in achieving gluteal symmetry. In lipoinjection, complications can occur up to $7 \%$ of cases [1]. Major complications include infection, donor site ischemia, pulmonary thromboembolism, fatty embolism, hematoma, and chronic seroma, while minor complications include accumulation of fluid in the donor area, paresthesia in the sciatic region, cellulitis, and gluteal fibrotic nodules [1]. In recent decades, the tendency has emerged for gluteal lipoinjection to replace gluteal augmentation with solid silicone implants due to the advantages of lipoinjection and the risk of complications associated with implant-based gluteal augmentation, even though implantation was traditionally the preferred technique [1].

The present case report describes a rare complication of gluteal augmentation with solid silicone implants, in which the implant was displaced from one gluteal pocket to the other. Displacement and rotation of the implant within a gluteal pocket has been reported in $0.59 \%$ of cases [6-8], but no reports in the literature describe a case in which two gluteal implants were lo-

\section{Fig. 1. Gluteal asymmetry with acute skin changes}

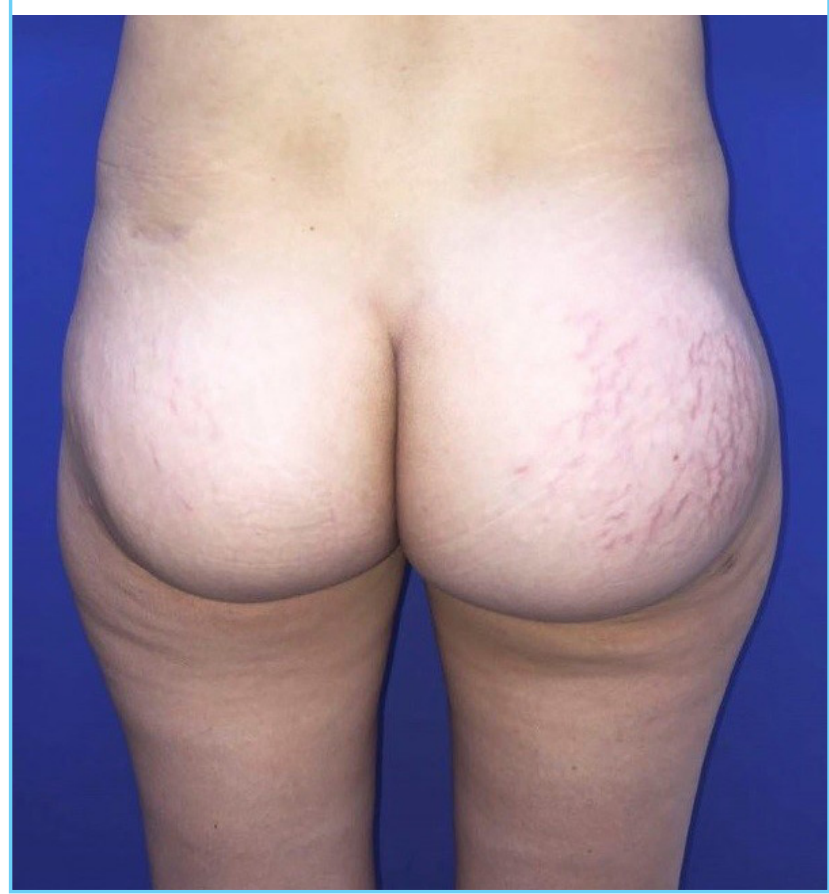

cated in a single pocket secondary to displacement; therefore, this case report makes a novel contribution.

\section{CASE}

The patient described herein was a 29-year-old woman who underwent gluteal augmentation with solid silicone implants on the border of Colombia and Venezuela in January 2016. Information from this procedure was unavailable. One week after surgery, the patient presented with a soft tissue infection with wound dehiscence and gluteal discharge; she underwent gluteal revision surgery with lavage, extraction and replacement of the gluteal implants, and antibiotic management. For 1-month, prolonged serous secretions continued and the patient received ongoing antibiotic therapy. Subsequently, her postoperative course apparently improved, and she remained asymptomatic for 8 months.

The patient presented for a consultation for a 3-day clinical course of progressive-onset pain in the right gluteus associated with changes in skin color and edema. There was no history of gluteal trauma (Fig. 1). In addition, the patient described rapidonset asymmetry of the buttocks. A physical examination confirmed that the left gluteus was flaccid and smaller than the contralateral side, while the right gluteus was larger, painful, and showed acute striae.

Nuclear magnetic resonance imaging was ordered, and showed displacement of the left gluteal implant into the right gluteal pocket, with the implants partially covered by the gluteal muscle (Fig. 2).

A surgical intervention was proposed for removal of the implants and delayed reconstruction after the conditions of the soft tissue improved; however, the patient did not agree to implant removal and wished for the implants to be repositioned.

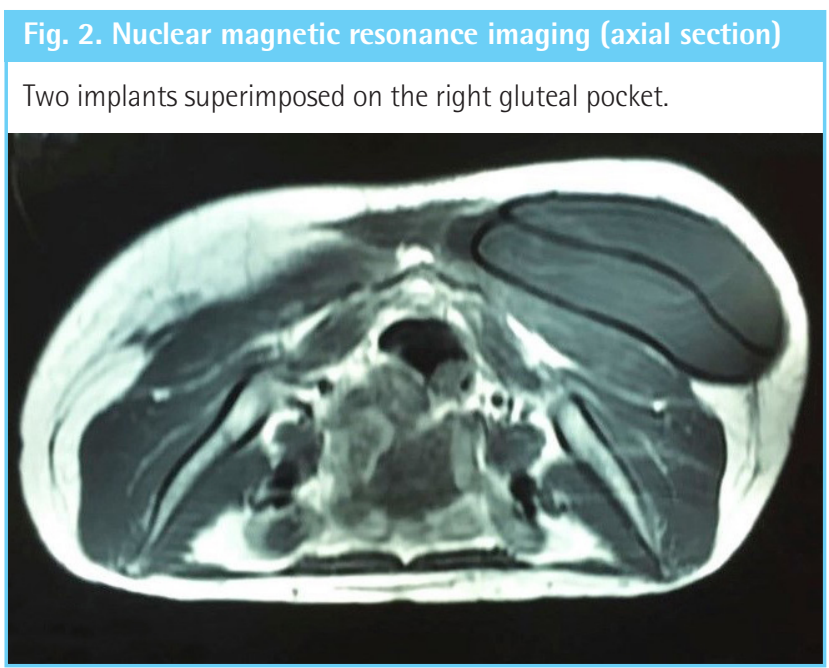


Therefore, it was decided to perform reconstructive surgery, after warning the patient of the risks. The operation was started by approaching the old incision in the gluteal cleft, where there was an absence of adhesion between the skin and the pre-sacral area. We then found two undamaged $350-\mathrm{mL}$ gluteal implants (Polytech) with rough surfaces. One was located above the other in the right gluteal pocket (Fig. 3). The left gluteal pocket had an extensive constriction, while the right pocket was enlarged. In addition, there was a large tunnel that communicated between the two pockets, and was covered by thickened capsular tissue (Fig. 3).

Subsequently, capsulotomy and enlargement of the left gluteal pocket in the lateral, superior, and inferior quadrants was done, followed by plication with Vicryl 0/0 sutures of the right pocket

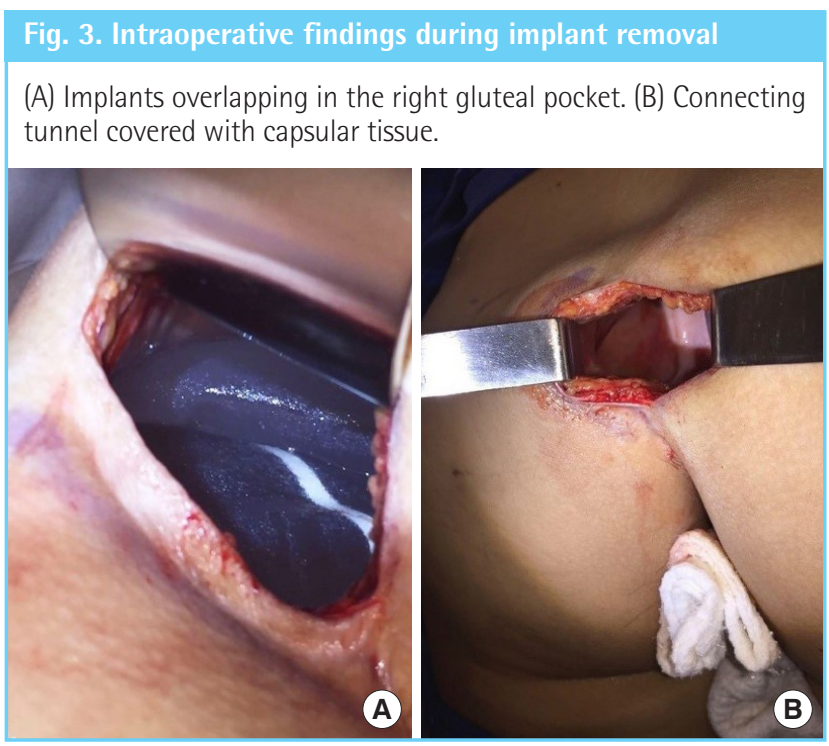

Fig. 4. Independent closure of the two gluteal pockets

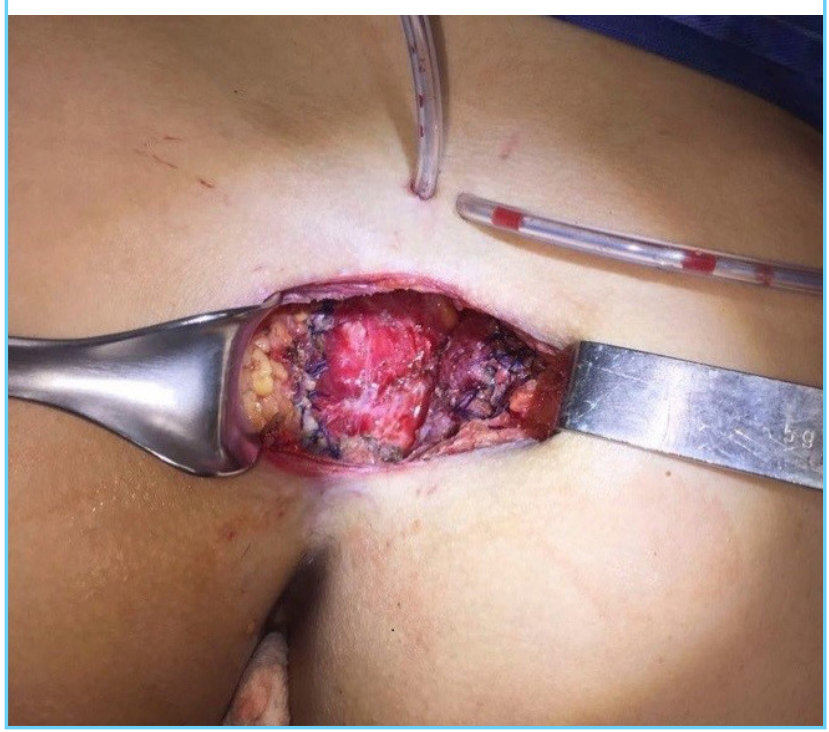

at the upper lateral quadrant. The implants and pockets were washed with abundant irrigation of iodine solution, and the implants were repositioned in the previously formed individualized pockets, making a closure in the following four planes along the middle line of the sacrum; (1) Plane 1: formed by carving capsular tissue flaps from the roof of the pocket's dome, as well as from the pre-sacral portion, and sutured with separate points using Vicryl 2/0; (2) Plane 2: formed by carving fatty fascial flaps from the buttocks, and sutured to the pre-sacral fascia with separate points using Vicryl 2/0; (3) Plane 3: formed by suturing the dermal plane to the deep plane with Vicryl 3/0; (4) Plane 4: skin closure on the gluteal cleft with mattress points made using Prolene 3/0.

A drain was left in each gluteal pocket for 15 days with careful recommendations for postoperative care on an outpatient basis. The patient experienced a good postoperative course with no associated complications (Fig. 4), and at a 5-month follow-up examination, we observed adequate positioning of the implants without malrotation, as well as a well-defined gluteal cleft with a high-quality scar (Fig. 5).

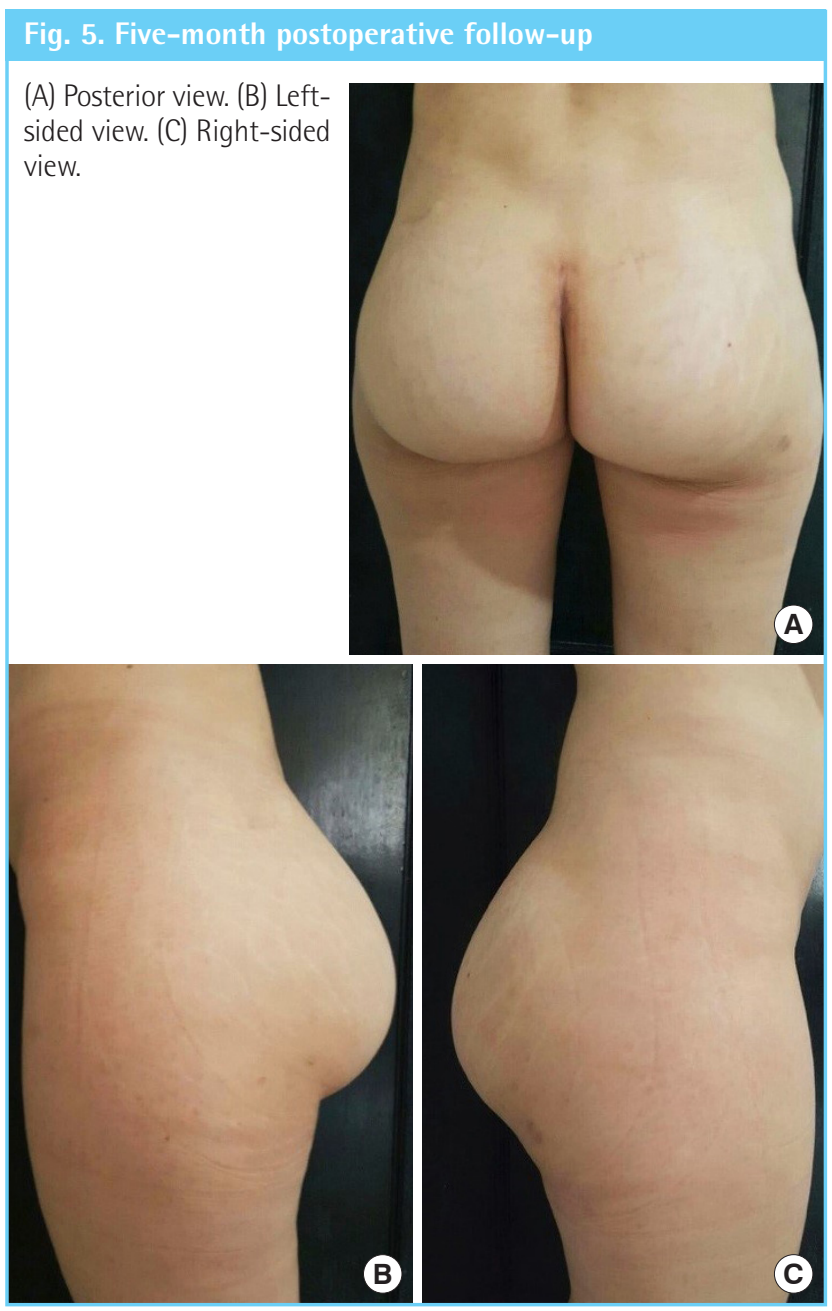




\section{DISCUSSION}

Gluteal augmentation with solid silicone implants has become significantly more common over the last two decades, increasing from 614 procedures in 2002 to 21,446 in 2014. The indications for this procedure include ptosis (especially in patients with major weight loss) and asymmetries in patients where there is insufficient fat for liposuction in the donor area $[1,2,5]$.

In a review, Senderoff [4] presented dehiscence, infection, seroma, implant extrusion, implant malrotation, and displacement of the implant as the most frequent complications of this procedure. Most of these complications are considered to be major, with a total complication rate reported in the literature of around 21.6\%. Most complications occur in the subfascial plane (62.1\%), followed by the submuscular plane (62.1\%) and the intramuscular plane (18.8\%).

In the intramuscular plane, common complications include dehiscence ( $30 \%$ of cases), seroma ( $2 \%$ to $4 \%$ ), and implant loss due to muscular dehiscence and exposure (2\% to $5 \%$, but reaching up to $30 \%$ in overweight patients or those with large implants). Other complications in this plane include asymmetry and lateral or superior migration of the implant depending on the configuration of the pocket or an incorrect implant position ( $3 \%$ to $5 \%$ ). Implant rupture is rare, as is capsular contracture (2\%). Transient sciatic paresthesia may occur in $20 \%$ of cases [1].

Most complications occur in the immediate postoperative period, although cases of late seromas have been reported several years after the procedure [9].

As part of the surgical technique, a unilateral or bilateral approach can be performed at the gluteal cleft in either the intramuscular or subfascial plane. Regardless of the plane, care should always be taken to preserve the tissue of the sacral fascia to achieve a good, tension-free closure. A disadvantage of the intramuscular plane is that dissection can be demanding and bleeding may occur.

The presence of undertreated hematomas can lead to dehiscence, infection, or the formation of a thick capsule. Fluid accumulation in the tissue as a result of dissection can also increase the risk of seroma formation (accumulation of fluid in the periprosthetic space), contamination, infection, and migration of the implant. Migration of the implant (generally to the lateral superior quadrant) can also result from muscular forces on intramuscular implants or overly extensive dissection of the intramuscular or subfascial plane. Lateral displacement may be caused by a seroma or untreated infection because the fluid can diffuse through the planes, and in extreme cases, exposure of the implant or extrusion can occur $[1,9]$.
In our case, two hypotheses may be proposed regarding the factors that led to this complication. First, poor surgical technique with insufficient tissue preservation to keep the two pockets separate, leading to the formation of a tunnel, in addition to very wide dissection of the pockets. Second, the presence of seroma and/or hematoma favoring infection, an inflammatory response, deterioration of the dissected soft tissues, and wound dehiscence associated with dissection of the planes caused by fluid accumulation, combining to cause displacement of the implant towards the contralateral side.

The patient did not want the implants to be removed. Since they were in good condition, the implants were repositioned and the two pockets were closed independently using four planes, obtaining satisfactory postoperative results without any new episode of implant displacement. However, problems may arise during long-term follow-up, even though the results were satisfactory at 5 months.

Although implant migration has been described in the literature, in our review we only found descriptions of lateral and superior displacement within the same gluteal pocket, making our case an atypical presentation.

Gluteal augmentation is a procedure that has become substantially more common over the past two decades. This procedure can be performed in the subfascial and/or intramuscular plane, although the intramuscular plane is most commonly used. As with every surgical procedure, it is not free of complications, with the most frequent complications including dehiscence, hematoma, seroma, infection, and malrotation and migration of the implant within the gluteal pocket (generally to the lateral quadrant). In our case, the atypical migration of a silicone implant from one gluteal pocket to the contralateral side was presented, with the presence of a treated infection as a predisposing factor. The surgical technique involved closure and separation of the buttock pockets in four planes, with satisfactory postoperative results and no recurrence. No similar case was found in our literature review [1-9].

\section{NOTES}

\section{Conflict of interest}

No potential conflict of interest relevant to this article was reported.

\section{Ethical approval}

The study was performed in accordance with the principles of the Declaration of Helsinki. Written informed consent was obtained. 


\section{Patient consent}

The patient provided written informed consent for the publication and the use of her images.

\section{Author contribution}

Conceptualization: JD Alviar Rueda. Data curation: JD Alviar Rueda, A Miranda-Diaz, A Gonzalez. Formal analysis: JD Alviar Rueda, A Miranda-Diaz, A Gonzalez, DC Navarro Leon. Methodology: JD Alviar Rueda, A Miranda-Diaz, A Gonzalez, DC Navarro Leon. Writing - original draft: JD Alviar Rueda, A Miranda-Diaz, A Gonzalez, DC Navarro Leon. Writing - review \& editing: JD Alviar Rueda, A Miranda-Diaz. Approval of final manuscript: all authors.

\section{ORCID}

Juan Dario Alviar Rueda

Audrey Jose Miranda-Diaz

https://orcid.org/0000-0003-4837-424X

https://orcid.org/0000-0002-8624-983X

Adriana Gonzalez Cely

https://orcid.org/0000-0002-4558-1801

Diana Carolina Navarro Leon

https://orcid.org/0000-0001-6650-1500

\section{REFERENCES}

1. Bruner TW, Roberts TL 3rd, Nguyen K. Complications of buttocks augmentation: diagnosis, management, and pre- vention. Clin Plast Surg 2006;33:449-66.

2. Mofid MM, Gonzalez R, de la Pena JA, et al. Buttock augmentation with silicone implants: a multicenter survey review of 2226 patients. Plast Reconstr Surg 2013;131:897901.

3. Flores-Lima G, Eppley BL, Dimas JR, et al. Surgical pocket location for gluteal implants: a systematic review. Aesthetic Plast Surg 2013;37:240-5.

4. Senderoff DM. Buttock augmentation with solid silicone implants. Aesthet Surg J 2011;31:320-7.

5. Sinno S, Chang JB, Brownstone ND, et al. Determining the safety and efficacy of gluteal augmentation: a systematic review of outcomes and complications. Plast Reconstr Surg 2016;137:1151-6.

6. Serra F, Aboudib JH, Neto JI, et al. Volumetric and functional evaluation of the gluteus maximus muscle after augmentation gluteoplasty using silicone implants. Plast Reconstr Surg 2015; 135:533e-541e.

7. Serra F, Aboudib JH. Gluteal implant displacement: diagnosis and treatment. Plast Reconstr Surg 2014;134:647-54.

8. Mezzine H, Khairallah G, Abs R, et al. Buttocks enhancement using silicone implants: a national practices assessement about 538 patients. Ann Chir Plast Esthet 2015;60: 110-6.

9. Senderoff DM. Aesthetic surgery of the buttocks using implants: practice-based recommendations. Aesthet Surg J 2016;36:559-76. 